\title{
Optimization of Flexible Flapping-Wing Kinematics in Hover
}

\author{
A. Gogulapati* \\ Ohio State University, Columbus, Ohio 43210 \\ and \\ P. P. Friedmann $₫$ and J. R. R. A. Martins \\ University of Michigan, Ann Arbor, Michigan 48109 \\ DOI: $10.2514 / 1 . J 053083$
}

\begin{abstract}
Hover-capable flapping-wing micro air vehicles are well suited for missions in confined spaces. The best design practices for flapping wings and their kinematics are largely unknown, especially for flexible wings. To address this issue, numerical optimization is applied to the design of the kinematics and structural sizing of a flapping wing using a surrogate-based approach. The surrogates are generated using kriging interpolation of the time-averaged thrust generated and power required by the wings. The thrust and power data are computed using a nonlinear approximate aeroelastic model developed in previous studies by the authors. A numerical optimization algorithm is used to identify designs that produce the desired combination of thrust and power. The design variables consist of parameters describing the flap-pitch kinematics and the stiffness of the flexible wings. A trend study of thrust and power indicate that the phase angle between flap and pitch motions significantly affects the wing performance when the stroke amplitudes and the frequency are fixed. Smaller amounts of pitch actuation produced peak thrust in flexible wings when compared to the rigid wings. Several flexible configurations produce higher thrust when compared to the best thrust-producing rigid configuration. However, rigid wings have higher propulsive efficiency when compared to flexible wings for the same amount of generated thrust. Thus, the actual design of a flapping wing will depend on the relative importance given to thrust production and propulsive efficiency.
\end{abstract}

\author{
Nomenclature \\ $=$ distance between pitching axis and midchord \\ of the airfoil \\ $=$ spatial correlation function \\ $=$ root chord \\ $=$ elastic modulus \\ $=$ unit vector along $Z_{\mathrm{SP}}$, resolved in the \\ $\left(X_{\mathrm{SP}}, Y_{\mathrm{SP}}, Z_{\mathrm{SP}}\right)$ coordinate system. \\ $=$ error \\ $=$ global mean in the surrogate \\ $=$ flapping frequency \\ $=$ indices in surrogate modeling \\ $=$ shape parameters in flap and pitch \\ $=$ number of design variables \\ $=$ number of sample points \\ $=$ number of test points \\ $=$ instantaneous unit normal vector at a point on \\ wing, resolved in the $\left(X_{\mathrm{SP}}, Y_{\mathrm{SP}}, Z_{\mathrm{SP}}\right)$ coordi- \\ nate system \\ $=$ aerodynamic power \\ $=$ local static pressure on the airfoil \\ $=$ radial location of the $j$ th spanwise section \\ with respect to the wing root
}

$A_{w} \quad=$ wing area

$a$

$C_{\mathrm{krg}}$

$c_{r}$

$E$

$\hat{\boldsymbol{e}}_{Z_{\mathrm{SP}}}$

$e$

$F$

$f$

$j, k$

$K_{\Phi}, K_{\alpha}$

$N_{\mathrm{dv}}$

$N_{\mathrm{sp}}$

$N_{\text {test }}$

$\hat{\boldsymbol{n}}_{\mathrm{SP}}$

$P$

$p$
$R$
Presented as Paper 2013-1646 at the 54th AIAA/ASME/ASCE/AHS/ASC Structures, Structural Dynamics, and Materials Conference, Boston, MA, 8 11 April 2013; received 16 September 2013; revision received 18 April 2014; accepted for publication 23 April 2014; published online 30 June 2014. Copyright $(\odot 2014$ by A. Gogulapati, P. P. Friedmann, and J. R. R. A. Martins. Published by the American Institute of Aeronautics and Astronautics, Inc., with permission. Copies of this paper may be made for personal or internal use, on condition that the copier pay the $\$ 10.00$ per-copy fee to the Copyright Clearance Center, Inc., 222 Rosewood Drive, Danvers, MA 01923 ; include the code 1533-385X/14 and \$10.00 in correspondence with the CCC.

*Postdoctoral Research Fellow, Department of Mechanical and Aerospace. Member AIAA.

${ }^{\dagger}$ François-Xavier Bagnoud Professor, Department of Aerospace Engineering. Fellow AIAA.

${ }^{¥}$ Associate Professor, Department of Aerospace Engineering. Associate Fellow AIAA.

\begin{tabular}{|c|c|c|}
\hline$R_{\text {span }}$ & $=$ & $\begin{array}{l}\text { span of the wing, measured from root to } \\
\text { the tip }\end{array}$ \\
\hline$s$ & $=$ & $\begin{array}{l}\text { approximation of the local mean-squared } \\
\text { error at } x \text { obtained from kriging }\end{array}$ \\
\hline$T$ & $=$ & thrust \\
\hline$t$ & $=$ & time \\
\hline$t_{\text {lespar }}$ & $=$ & thickness of leading-edge spar \\
\hline$t_{\text {rib1 }}-t_{\text {rib5 }}$ & $=$ & thickness of the ribs (battens) \\
\hline$U_{\text {ref }}$ & $=$ & reference speed \\
\hline$U_{\text {tip }}$ & $=$ & maximum tip speed \\
\hline$w$ & $=$ & $\begin{array}{l}\text { parameter used to balance the search process } \\
\text { in the efficient global optimization }\end{array}$ \\
\hline$\left(X_{\mathrm{SP}}, Y_{\mathrm{SP}}, Z_{\mathrm{SP}}\right)$ & $=$ & coordinate system fixed to the stroke plane \\
\hline$\left(X_{w}, Y_{w}, Z_{w}\right)$ & $=$ & wing-fixed coordinate system \\
\hline$x$ & $=$ & vector of design variables \\
\hline$x_{\mathrm{SP}}$ & $=$ & $\begin{array}{l}\text { instantaneous position vector of a point on the } \\
\text { wing in the }\left(X_{\mathrm{SP}}, Y_{\mathrm{SP}}, Z_{\mathrm{SP}}\right) \text { coordinate system }\end{array}$ \\
\hline $\boldsymbol{x}_{w}$ & $=$ & $\begin{array}{l}\text { instantaneous position vector of a point on the } \\
\text { wing in the }\left(X_{w}, Y_{w}, Z_{w}\right) \text { coordinate system }\end{array}$ \\
\hline$y$ & $=$ & function to be approximated \\
\hline$\hat{y}_{\mathrm{krg}}$ & $=$ & kriging prediction \\
\hline$y_{\min }$ & $=$ & current best value of the objective function \\
\hline$Z$ & $=$ & $\begin{array}{l}\text { deviation about the global mean, assumed to } \\
\text { be a stochastic process }\end{array}$ \\
\hline$\alpha$ & $=$ & pitch angle \\
\hline$\alpha_{1}$ & $=$ & pitch amplitude \\
\hline$\beta$ & $=$ & constant \\
\hline$\vartheta_{k}, \varsigma_{k}$ & $=$ & correlation parameters in kriging \\
\hline$\nu_{\infty}$ & $=$ & kinematic viscosity of the fluid \\
\hline$\nu$ & $=$ & Poisson's ratio \\
\hline$\rho$ & $=$ & density of the material \\
\hline$\rho_{\infty}$ & $=$ & freestream density of the fluid \\
\hline$\Phi$ & $=$ & flap angle \\
\hline$\Phi_{1}$ & $=$ & flap amplitude \\
\hline$\phi_{\text {dist }}$ & $=$ & normal cumulative distribution function \\
\hline$\phi_{\mathrm{den}}$ & $=$ & normal probability density function \\
\hline$\varphi_{\alpha}$ & $=$ & phase angle between flap and pitch motions \\
\hline
\end{tabular}

\section{Introduction}

H OVER-CAPABLE flapping-wing micro air vehicles (FWMAVs) are expected to be suitable for missions involving 
confined spaces carried out at low altitudes and short ranges, such as search inside buildings. The expected maximum dimension of such vehicles is less than $15 \mathrm{~cm}$, and their weight is less than $100 \mathrm{~g}$. Such vehicles operate at low Reynolds numbers $\left(10^{2}<R e<10^{4}\right)$ and low forward flight speed (less than $15 \mathrm{~m} / \mathrm{s}$ ) [1].

The wing kinematics of hover-capable flyers consists predominantly of flapping and pitching motions, combined with a comparatively small elevation motion [1]. Research on flapping wings [1 $1-5]$ indicates that the aerodynamic forces vary significantly in the design space described by the kinematic parameters. The power available for FWMAVs is also limited due to the restrictions on size and weight [6]. Thus, the selection of kinematics that produces the best combination of thrust and power is an important consideration for the development of wings intended for FWMAVs.

Optimization of the kinematics for rigid and flexible wings was considered in [7-9]. In [7], a semi-empirical aerodynamic model was combined with a global optimization algorithm to identify powerminimizing kinematics for several rigid bio-inspired wing configurations in hover undergoing combined flap-pitch motion. Optimal kinematics were found to resemble the kinematics in the corresponding biological flyers. In [8], the lift and propulsive efficiency (lift generated per unit power) of rigid airfoils undergoing pitchplunge motions was simulated using aerodynamics based on the solution of the Navier-Stokes equations. The performance in forward flight was optimized using a gradient-based (GB) approach. In [9]], optimal combinations of wing kinematics, flexibility, and planform were identified using a GB optimization approach. The wings, modeled as beams, were actuated by a combined flap-pitchelevation motion. The aerodynamic loads and power were computed using a quasi-steady formulation. Configurations that reduced the required power were identified.

In the current study, the aerodynamic force and power of the flapping wing are computed using an approximate aeroelastic model developed by the authors $[10,11]$. The model produced acceptable correlation with computational fluid dynamics (CFD) simulations and experiments for a range of kinematic, structural, and aerodynamic parameters [10-12]. It also yields a significant reduction in computational cost compared to CFD-based approaches. However, the cost of the large number of simulations required in an optimization study prevents the direct coupling of the aeroelastic analysis with the optimization algorithm. Therefore, surrogate models, which represent computationally efficient global approximations of the analysis [13], are used. The surrogates are constructed by interpolating the output obtained from a suitable number of true function evaluations and are used to replace the expensive computations when exploring the design space.

Previous studies on flapping-wing studies employing surrogate approaches were limited to rigid wings [14]. Surrogates generated from CFD data were used to study the influence of leading-edge vortices (LEVs) and tip vortices on the lift and thrust generated by rectangular wings undergoing prescribed translation and pitching motions. Regions in the design space where tip vortices had a beneficial effect were identified. Surrogate-based approaches have been shown to have considerable potential for optimizing nonlinear aeroelastic systems. In $[15,16]$, surrogate-based optimization was used to design helicopter blades with low vibration levels in forward flight. Surrogates based on kriging were found to be very effective for predicting the vibratory hub loads in forward flight.

The overall goal of this paper is to identify combinations of wing kinematics and flexibility that enhance the performance for anisotropic flapping wings in hover. The performance metric is represented by the thrust generated and the power consumed by the wings. The specific objectives of this paper are to develop accurate surrogate models that predict the performance of rigid and anisotropic flapping wings in hover, and compare the performance of rigid and flexible wings in a large parameter space to assess the effect of wing flexibility.

The remainder of the paper is organized as follows. First, the nonlinear approximate aeroelastic model used to calculate the thrust and power generated by the flapping wings, and the surrogatebased optimization procedure is summarized. Next, the optimization studies conducted using rigid and flexible wings are described. Finally, the important conclusions gleaned from the results are presented.

\section{Overview of the Nonlinear Aeroelastic Model}

The aeroelastic model is obtained by coupling a nonlinear finiteelement-based structural dynamic model of the wing with an approximate unsteady aerodynamic model. The model, developed in previous studies by the authors $[10,11]$, exhibits acceptable correlation with CFD simulations and experiments for a wide range of kinematic, structural, and aerodynamic parameters [10-12]. The components of the aeroelastic model and the corresponding validation and verification studies are described in [10-12]. Therefore, the discussion presented in this paper is limited to an overview.

\section{A. Structural Dynamic Model and Wing Kinematics}

The wings are modeled in MSC MARC, a commercially available nonlinear finite-element code [17]. The structural dynamic model is based on thin shell triangular elements, shown in Fig. 1a, suitable for modeling wings undergoing large rigid-body rotations and moderateto-large flexible deformation due to coupled bending and twisting in both the spanwise and the chordwise directions. Each node has six degrees of freedom: three displacements and three rotations. The nodal degrees of freedom are interpolated using a bilinear interpolation.

The wings are actuated by combined flap-pitch motions that are based on the kinematics considered in [7]. The flapping and pitching motions, given by

$$
\Phi(t)=\Phi_{1} \frac{\sin ^{-1}\left[K_{\Phi} \sin (2 \pi f t)\right]}{\sin ^{-1}\left(K_{\Phi}\right)}
$$

and

$$
\alpha(t)=-\alpha_{1} \frac{\tanh \left[K_{\alpha} \sin \left(2 \pi f t+\varphi_{\alpha 1}\right)\right]}{\tanh \left(K_{\alpha}\right)}
$$

are prescribed about the flapping and feathering axes shown in Fig. 1a. The variables $K_{\Phi}$ and $K_{\alpha}$ govern the shape of the flap and pitch kinematic patterns as shown in Fig. 2. The movement of the feathering axis due to the time-dependent flapping motion describes the stroke plane (SP), shown in Fig. 1b. The axes $X_{w}$ and $Y_{w}$, shown in Fig. 1a, are assumed to coincide with $X_{\mathrm{SP}}$ and $Z_{\mathrm{SP}}$, shown in Fig. 1b, respectively, at the start of the motion. The wing rotations are converted to displacements using the following relation:

$$
\boldsymbol{x}_{\mathrm{SP}}=\left[\begin{array}{ccc}
\cos \Phi & 0 & \sin \Phi \\
0 & 1 & 0 \\
-\sin \Phi & 0 & \cos \Phi
\end{array}\right] \cdot\left[\begin{array}{ccc}
1 & 0 & 0 \\
0 & \cos \alpha & -\sin \alpha \\
0 & \sin \alpha & \cos \alpha
\end{array}\right] \cdot \boldsymbol{x}_{w}
$$

and applied as displacement boundary conditions at the wing root.

\section{B. Unsteady Aerodynamic Model}

The unsteady aerodynamic model $[10,11]$ is a modification of the original model described in $[\underline{18}, 19]$. The formulation incorporates LEVs, a wake model, and accounts for the effects of wing flexibility, forward flight, and fluid viscosity. The model is based on twodimensional (2-D) potential flow and is applied on the wing in a striptheory manner. Separation of the flow from the leading edge (LE) is assumed (i.e., it is either present or not, along with the location of the separation point). The model $[10,11]$ does not capture spanwise flow and tip vortices. The accuracy of the approach is expected to decrease when such effects have a predominant effect of the forces. The aerodynamic model exhibits reasonable agreement with CFD-based aerodynamics and experiments in both the time-averaged values and the time histories of the forces for the range of kinematic and aerodynamic parameters considered in the current work [10-12]. 


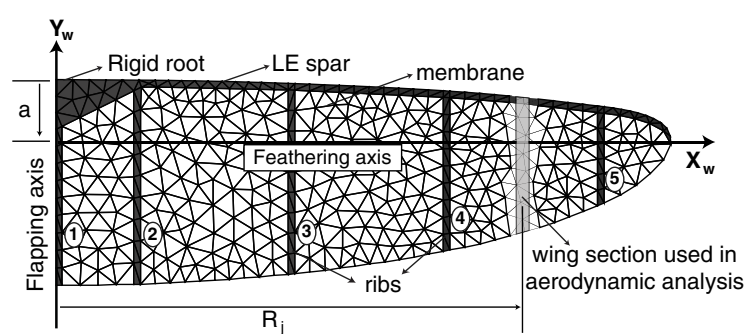

a) Zimmerman wings with wing-fixed coordinate system

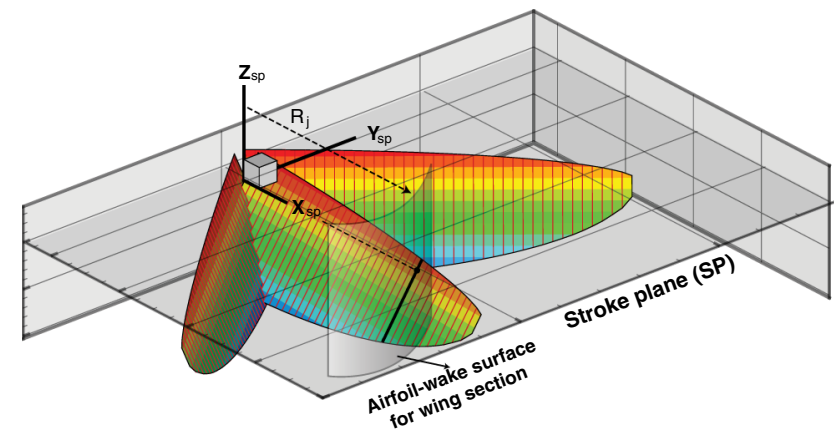

b) Stroke plane

Fig. 1 Wing configuration and coordinate systems.
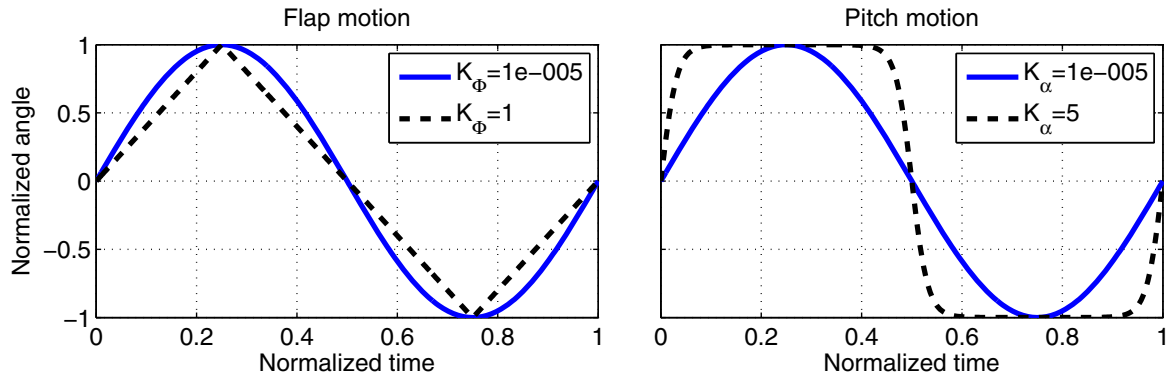

Fig. 2 Variation of flap and pitch kinematic patterns with shape parameters.

Thus, it is suitable for the optimization studies conducted in this paper.

The overall approach [10] is illustrated in Fig. 3. The wing is divided into several spanwise stations, shown in Fig. 1a, where each section is represented as an airfoil. For each airfoil, the airfoil motion and the shed wake geometry are approximated using an airfoil-wake surface that is represented by a cylinder normal to the stroke plane (SP), shown in Fig. 1b. The cylindrical surface is straightened out

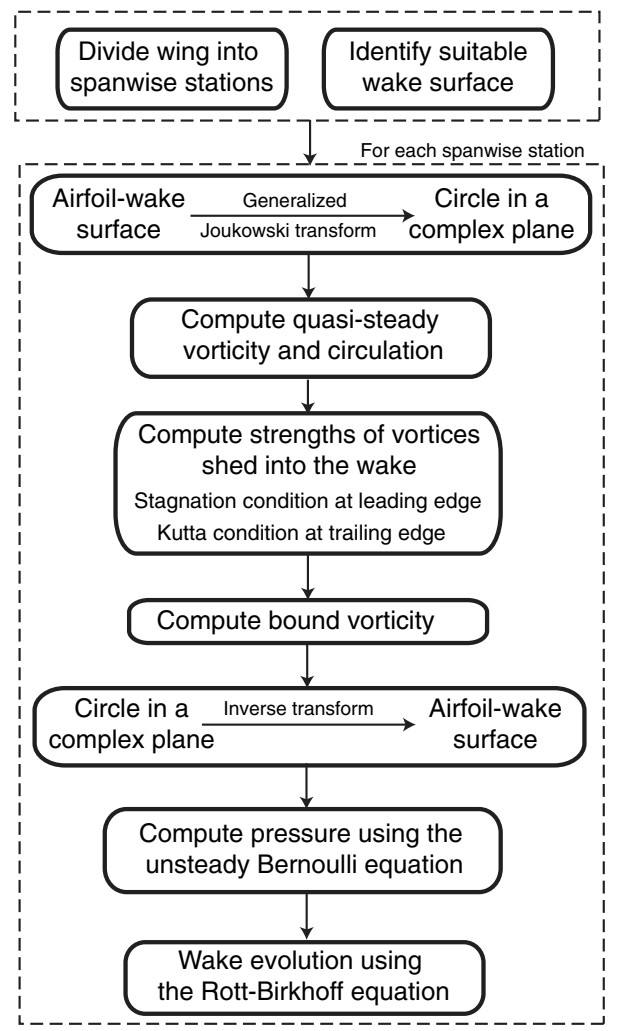

Fig. 3 Computational procedure for the aerodynamic model. (unwrapped) to represent a 2-D plane. The airfoil and shed vortices are modeled in this plane by transforming the airfoil into a circle on a complex plane using a conformal mapping. The bound and shed wake vorticity are computed on the complex plane. The quasi-steady component of vorticity is obtained by neglecting the effect of the shed wake. The strength of shed vorticity is computed by enforcing a stagnation condition at the leading edge (LE) and a Kutta condition at the trailing edge. The airfoil bound vorticity is obtained as a sum of the quasi-steady and wake-induced vorticity on the airfoil. Next, the vorticity on the complex plane is transformed back to the airfoil-wake surface using an inverse transform. Subsequently, the unsteady aerodynamic pressure on the surface of the wing is computed from the total vorticity using the unsteady Bernoulli equation. Finally, the shed vorticity is convected using the Rott-Birkhoff equation, derived from Biot-Savart law for 2-D flow.

The SP is assumed to be horizontal for the case of hover. The thrust is defined as the component of the aerodynamic force normal to the $\mathrm{SP}$ (i.e., along the $Z_{\mathrm{SP}}$ axis) and is given by

$$
T(t)=\iint_{A_{w}} \hat{\boldsymbol{e}}_{Z_{\mathrm{SP}}} \cdot\left(p \mathrm{~d} A_{w} \hat{\boldsymbol{n}}_{\mathrm{SP}}\right)
$$

The aerodynamic power, which is the component of the total mechanical power required to overcome the aerodynamic forces acting on the wing, is calculated from the instantaneous wing velocity and pressure distributions as

$$
P(t)=\iint_{A_{w}} \dot{\boldsymbol{x}}_{\mathrm{SP}}(t) \times\left(p \mathrm{~d} A_{w} \hat{\boldsymbol{n}}_{\mathrm{SP}}\right)
$$

The time-averaged (or mean) thrust and aerodynamic power, which are the performance metrics used in the current study, represent the average payload capacity and power penalty over a flapping cycle, respectively. The wing performance is enhanced by increasing the payload capacity and decreasing the power penalty. 


\section{Fluid-Structure Coupling}

The equations of motion for the aeroelastic problem are obtained in MARC using an updated Lagrangian method [20]. Time-domain integration of the equations is done using a single-step Houbolt numerical scheme [21]. The fluid-structure coupling in MSC MARC is accomplished by two user-defined subroutines: FORCDT and FORCEM. The aeroelastic model is illustrated by the block diagram shown in Fig. 4. At each time step, rigid-body motion is prescribed by specifying the displacements at the nodes using the FORCDT subroutine. The aerodynamic loads are computed based on the wing motion at the beginning of each time step and applied as pressure on the structure using the FORCEM subroutine. This subroutine is called from the main program for each step of the Newton-Raphson iteration within a time step to ensure convergence of the structural displacements. Finally, the vortices shed into the wake are convected at the end of the time step. Additional details are provided in [22].

\section{Surrogate Modeling and Optimization}

Surrogate models are used to predict the time-averaged aerodynamic force and power as functions of the kinematic and structural parameters. The surrogate modeling approach used is similar to [16] and is summarized next.

\section{A. Generation of the Surrogate and Testing Its Accuracy}

The steps required for surrogate generation are illustrated in Fig. 5. The initial set of surrogates are constructed using fitting points selected to sample the parameter space. These points are selected using an optimal Latin hypercube (OLH) sampling [13]. In this approach, the design variables are partitioned into several equally spaced sections, thereby dividing the parameter space into several equally sized hypercubes. A spreading criterion is used ensure uniform sampling in the design space. The accuracy of the surrogates is estimated at a set of testing points that are not included in the set of fitting points. The testing points are also generated using an OLH sampling to represent the parameter space in an efficient manner.

The aerodynamic or aeroelastic simulations are performed at each of the design points. The simulations are run in parallel on several

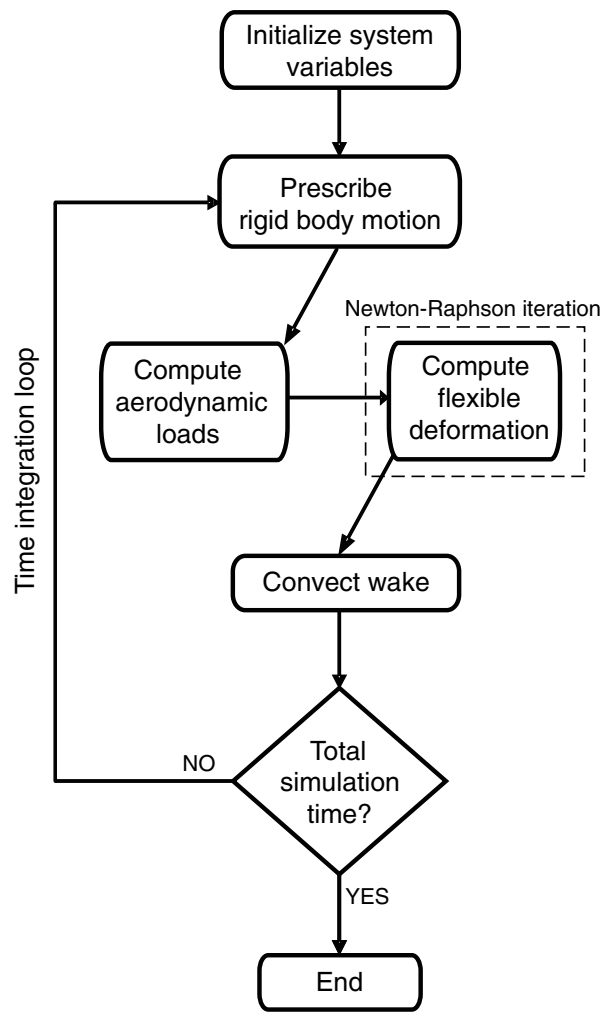

Fig. 4 Computational framework for the aeroelastic model in MARC.

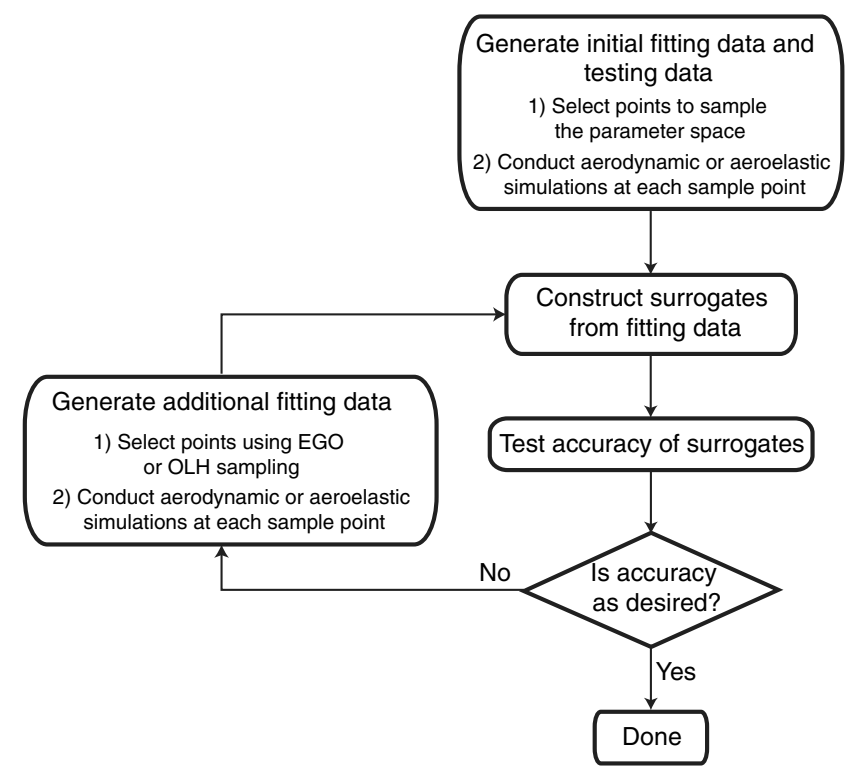

Fig. 5 Steps in surrogate modeling.

processors because the true function evaluation at each design point can be obtained independently of the evaluations at other design points.

The surrogates are created from the fitting data following the kriging interpolation approach used in [16]. In kriging, the unknown function is approximated by

$$
y(x)=F(x)+Z(x)
$$

where $F(x)$ is the global mean, and $Z(x)$ is the deviation about the global mean, represented as a stochastic process. Previous studies $[16,23,24]$ indicate that using $F(\boldsymbol{x})=\beta$, where $\beta$ is constant, is adequate for producing approximations of sufficient accuracy. Therefore, in this study, it is assumed that $F(x)=\beta$. A crucial step in determining $Z(\boldsymbol{x})$ is the selection of a spatial correlation function (SCF), which accounts for the effect of each interpolation point on every other point. The SCF used in this study is given by a generalized Gaussian function [23] as

$$
C_{\mathrm{krg}}\left(\boldsymbol{x}^{(i)}, \boldsymbol{x}^{(j)}\right)=\exp \left[\sum_{k=1}^{N_{\mathrm{dv}}} \vartheta_{k}\left|\boldsymbol{x}_{k}^{(i)}-\boldsymbol{x}_{k}^{(j)}\right|^{\varsigma_{k}}\right]
$$

where $\vartheta_{k}$ and $\varsigma_{k}$ determine the quality of the fit. The general procedure used to identify $\vartheta_{k}$ and $\varsigma_{k}$, and subsequently $Z(x)$, is described in [25].

The accuracy of the surrogates is improved by adding new points to the set of existing fitting points. Two approaches are used to determine the new points. In the first approach, additional fitting points are determined in an ad hoc manner using an OLH sampling for specific regions of the parameter space where the accuracy of the surrogate is found to be inadequate. In the second approach, the accuracy of the surrogates is improved in a systematic manner by using an efficient global optimization (EGO) algorithm [16,26,27]. An expected improvement function (EIF) [26,27], defined based on the local error in prediction provided by the kriging approach, is used to identify regions of the parameter space where the predictions of the surrogate are unreliable due to a high amount of uncertainty. Subsequently, additional sample or "infill" points are identified by maximizing the EIF. Two types of EIFs can be defined:

$$
\mathrm{EIF}_{1}=\left(y_{\text {min }}-\hat{y}_{\mathrm{krg}}\right) \phi_{\text {dist }}\left(\frac{y_{\text {min }}-\hat{y}_{\mathrm{krg}}}{s}\right)
$$




$$
\mathrm{EIF}_{2}=s \phi_{\mathrm{den}}\left(\frac{y_{\min }-\hat{y}_{\mathrm{krg}}}{s}\right)
$$

where $s$ is obtained following [25]. The function $\mathrm{EIF}_{1}$ is large when $\hat{y}_{\mathrm{krg}}$ is likely to be better than $y_{\min }$. The term $\mathrm{EIF}_{2}$ is large in regions of the parameter space where the mean-squared error is large (i.e., there is much uncertainty in the surrogate prediction). The search process can be performed in regions of the parameter space where the designs are expected to be optimal (using only EIF $_{1}$ ) or where there is considerable uncertainty in surrogate prediction (using only $\mathrm{EIF}_{2}$ ). Alternately, a balance between the two types of searches is obtained by using a weighted expected improvement function (WEIF) [16] as

$$
W \mathrm{EIF}(\boldsymbol{x})= \begin{cases}w \mathrm{EIF}_{1}+(1-w) \mathrm{EIF}_{2} & \text { if } s>0 \\ 0 & \text { if } s=0\end{cases}
$$

where $0 \leq w \leq 1$. In the current study, additional points to increase accuracy of the surrogates were identified using $\mathrm{EIF}_{2}$ or by substituting $w=0$ in Eq. (5).

The OLH sampling of the parameter space is performed using the iSight software $[28,29]$. The surrogates are generated in MATLAB using the DACE toolbox [25]. The EGO algorithm is implemented in MATLAB using an in-house code.

\section{B. Optimization}

Numerical optimization is employed to achieve two separate goals: 1) locate the new sample points to improve the accuracy of the surrogates using the EGO algorithm, and 2) identify designs that yield the desired combination of aerodynamic force and power once surrogates of sufficient accuracy are generated. The optimum is identified by using a combination of global and gradient-based approaches in sequence. The design point predicted by the global search algorithm is used as the initial guess for the gradient-based algorithm. This hybrid approach reduces the computational cost of the optimization when the design points found by the global search are a sufficiently good starting guess for the gradient-based approach.

The global search is based on the GODLIKE (from "global optimum determination by linking and interchanging kindred evalua-

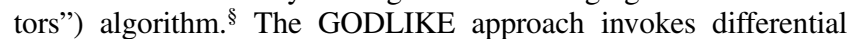
evolution [30], genetic algorithms [31], particle swarm optimization [32], and adaptive simulated annealing algorithms [33] and links them together by inserting random members of the population obtained from one approach into the population of other approaches during their optimization cycles. This interchange of the predictions from different algorithms eliminates weakness in the population due to the limitations of the individual approaches. The use of stronger populations in each cycle enhances the probability of locating a true global optimum. However, the interchange also adversely affects the convergence rate, thereby increasing the number of function evaluations needed for the optimization. Therefore, the GODLIKE algorithm is computationally less efficient but more robust compared to the individual constituents. The gradient-based approach uses an interior point algorithm [34-36], in which a constrained minimization problem is solved by replacing it by a sequence of approximate minimization problems. The inequality constraints are implemented using logarithmic penalty functions. In each iteration, the size of the optimization step is computed by solving the discretized version of the Karush-Kuhn-Tucker equations [34-36].

The GODLIKE and the interior-point algorithms are implemented in MATLAB using the GODLIKE toolbox and by invoking the fmincon function provided in the optimization toolbox, respectively.

\section{Results and Discussion}

The results obtained from the surrogate-based optimization of rigid and flexible wings in hover are for a Zimmerman planform,

${ }^{\S}$ Data available online at http://www.mathworks.com/matlabcentral/ fileexchange/24838-godlike-a-robust-single-multi-objective-optimizer [retrieved 6 June 2014]. shown in Fig. 1a, that has been used in previous studies $[10,11,37]$. These wings have $R_{\text {span }}=75 \mathrm{~mm}, c_{r}=25 \mathrm{~mm}$, and an aspect ratio of 7.65. The computations were carried out for air $\left(\rho_{\infty}=\right.$ $1.209 \mathrm{~kg} / \mathrm{m}^{3}$ and $\left.\nu_{\infty}=1.64 \times 10^{-5} \mathrm{~m}^{2} / \mathrm{s}\right)$. The approximate aerodynamic loads are calculated by dividing the wing into 10 spanwise stations, where a spanwise station is depicted in Fig. 1a. The calculations are performed by discretizing each airfoil using 101 elements, using four wake subiterations per time step, and a vortex core radius equal to $2.5 \%$ of the corresponding airfoil chord. Leading-edge flow separation is assumed throughout the flapping stroke. The aerodynamic loads are computed using the unsteady Bernoulli equation. For all the simulations, an approximate steady state was obtained after one cycle. Therefore, the time-averaged (or mean) quantities are calculated by averaging the corresponding time histories over cycles 2 through 5 .

\section{A. Results for Rigid Wings}

In previous studies employing the aerodynamic model [10,11], the simulations were conducted using 59 spanwise stations. In the current paper, only 10 spanwise stations are used to reduce the computational cost required to generate the fitting and testing data. The accuracy of the computations was evaluated by comparing the predictions using 10 spanwise stations to those obtained using 59 spanwise stations. The errors in thrust and power were calculated at 50 points obtained from an OLH sampling of the parameter space, for the allowed ranges of the kinematic design variables (DVs) listed in the Table 1 . The simulations at each sample point were performed

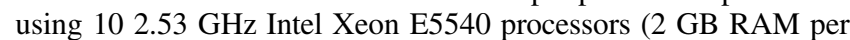
processor). The maximum times per simulation were approximately 1.5 and $9 \mathrm{~h}$ when using 10 and 59 spanwise stations, respectively. The maximum local errors in thrust and power are 6 and 3\% of the corresponding maximum values in the data sets. The rms errors in thrust and power are less than $2 \%$ of the corresponding maximum values. This implies that the accuracy achieved when using 10 spanwise stations is adequate.

Kriging surrogates to predict the mean thrust and mean power generated by rigid wings were obtained initially from a set of 200 points. The accuracy of the surrogates was tested at the set of $50 \mathrm{OLH}$ points obtained previously. The mean and maximum errors in thrust and power are defined as

$$
e_{\max }=\max \left\{e_{j}\right\}
$$

and

$$
e_{\text {mean }}=\frac{1}{N_{\text {test }}} \sum_{j=1}^{N_{\text {test }}} e_{j}
$$

where

$$
e_{j}=\frac{\left|\hat{y}_{\mathrm{krg}}^{j}-y^{j}\right|}{\max \left\{y^{j}\right\}}
$$

where $y_{\mathrm{krg}}^{j}$ and $y^{j}$ are the surrogate prediction and true function evaluation at the $j$ th test point. Next, the accuracy of the surrogates was improved by adding 149 new fitting points selected as follows. First, the initial surrogate was used in the EGO algorithm to identify

Table 1 Design variables and their bounds

\begin{tabular}{lc}
\hline \hline Description & Bounds \\
\hline Flap amplitude & $10 \leq \Phi_{1} \leq 60 \mathrm{deg}$ \\
Pitch amplitude & $0 \leq \alpha_{1} \leq 60 \mathrm{deg}$ \\
Phase amplitude & $0 \leq \Phi_{\alpha 1} \leq 360 \mathrm{deg}$ \\
Shape parameter in flap & $0 \leq K_{\Phi} \leq 1$ \\
Shape parameter in pitch & $0 \leq K_{\alpha} \leq 5$ \\
Location of pitch axis & $0 \leq a \leq 0.25 c_{r}$ \\
Flapping frequency & $10 \leq f \leq 30 \mathrm{~Hz}$ \\
\hline \hline
\end{tabular}


Table 2 Errors in thrust

\begin{tabular}{lcrr}
\hline \hline Errors & 197 points & 346 points & 1215 points \\
\hline \multicolumn{4}{c}{ Mean and maximum errors } \\
\hline Mean, \% & 6.3 & 5.9 & 2.7 \\
Maximum, \% & 52 & 72 & 14.5 \\
& Distribution of errors \\
\hline$>20 \%$ & 4 points & 1 point & 0 point \\
$<20 \%$ & 46 points & 49 points & 50 points \\
\hline \hline
\end{tabular}

50 points. The surrogate was improved by incorporating the new fitting points, and a second path through EGO was used to identify 50 points. The process was repeated for a third time to select 49 additional points. In the iterations using EGO, each new point was picked so that the minimum normalized distance between the point and all other preceding points was a user specified value of 0.1 . The errors in the surrogates are summarized in Tables 2 and 3 . Large errors were found to occur at the boundaries corresponding to $\alpha_{1}=0 \mathrm{deg}$, and $\left\{\Phi_{1}=60 \mathrm{deg}, f=30 \mathrm{~Hz}\right\}$. Therefore, 870 additional points were introduced using a combination of OLH samplings and EGO as follows. In the first sampling, $100 \mathrm{OLH}$ points were generated for $\alpha_{1}=0 \mathrm{deg}, 10 \leq \Phi_{1} \leq 62.5 \mathrm{deg}$ and $10<f<32.5 \mathrm{~Hz}$, where the ranges for other DV are given in Table 1. Next, additional 290 OLH points were introduced for $52.5 \leq \Phi_{1}{ }^{-} \leq 62.5 \mathrm{deg}$ and $27.5<f<$ $32.5 \mathrm{~Hz}$, where the ranges for other DV are given in Table 1 . Next, 290 points were selected using EGO for $50 \leq \Phi_{1} \leq 60 \overline{\mathrm{deg}}$ and $20<f<30 \mathrm{~Hz}$, where the ranges for other DV are given in Table 1 . Finally, two sets of 195 OLH points were generated for $\Phi_{1}=$ $55 \mathrm{deg}, f=25 \mathrm{~Hz}$ and $\Phi_{1}=60 \mathrm{deg}, f=30 \mathrm{~Hz}$, respectively. Introduction of the additional points resulted in a significant reduction in the maximum error in thrust, as evident from Table 2. The reduction of maximum error in power was relatively smaller, as shown in Table 3 . The distribution of errors are provided in Tables 2 and 3 . The results in Tables 2 and 3 indicate that the surrogates have acceptable accuracy (errors less than $20 \%$ ) over a large region of the parameter space.

The surrogates are combined with the numerical optimization algorithm to identify kinematics that maximized the thrust. The kinematic variables corresponding to the maximum thrust based on the surrogates as well as the true function evaluations are shown in Tables 4 and 5. The relative errors in thrust and power prediction are 3 and $8 \%$, respectively. Note that the maximum thrust in the set of fitting points is $5.6 \mathrm{~g}$. Thus, the optimum predicted using the surrogate-based optimization approach produces higher thrust compared to the best thrust producing design in the set of fitting points.

Table 3 Errors in power

\begin{tabular}{lrrr}
\hline \hline Errors & 197 points & 346 points & 1215 points \\
\hline \multicolumn{4}{c}{ Mean and maximum errors } \\
\hline Mean, \% & 3.5 & 2.4 & 1.5 \\
Maximum, \% & 38.7 & 27 & 20.5 \\
& Distribution of errors \\
\hline$>20 \%$ & 1 point & 1 point & 1 point \\
$<20 \%$ & 49 points & 49 points & 49 points \\
\hline \hline
\end{tabular}

Table 4 Design point corresponding to maximum thrust: kinematic design variables

\begin{tabular}{lc}
\hline \hline Parameter & Value \\
\hline$\Phi_{1}$ & $60 \mathrm{deg}$ \\
$\alpha_{1}$ & $50 \mathrm{deg}$ \\
$\phi_{\alpha 1}$ & $80.2 \mathrm{deg}$ \\
$K_{\Phi}$ & 0.183 \\
$K_{\alpha}$ & 3.65 \\
$a$ & 0.14 \\
$f$ & $30 \mathrm{~Hz}$ \\
\hline \hline
\end{tabular}

Table 5 Design point corresponding to maximum thrust: surrogate predictions and true function evaluations

\begin{tabular}{lcc}
\hline \hline & Surrogate prediction & True function evaluation \\
\hline$T_{\max }, \mathrm{g}$ & 5.89 & 5.75 \\
$P_{T_{\max }}, \mathrm{W}$ & 0.482 & 0.452 \\
\hline \hline
\end{tabular}

The variation of the thrust and power with the various DV, calculated for the kinematics that produce maximum thrust, are shown in Fig. 6. The value of the kinematic parameter corresponding to maximum thrust is indicated by a circle. Both the thrust and power increase monotonically with increasing flap amplitude, as shown in Fig. 6a. This is due to the fact that thrust and power are competing design objectives. Figure $6 \mathrm{~b}$ shows that thrust and power are nonmonotonic with pitch amplitude, indicating that only a limited amount of pitching motion is beneficial. The phase angle affects the interaction of the wing with the vortices generated at the end of the previous stroke. This effect, termed as the wake capture [4], is an unsteady mechanism that is not captured by aerodynamic formulations that use quasi-steady or fixed-wake assumptions. The thrust and power vary significantly over the range of phase angles considered, as shown in Fig. 6c. This implies that the wake-capture mechanism can be used to tailor the thrust and power of a flapping wing without significant modification of the stroke amplitude or flapping frequency. The effect of the shape parameters given in Eqs. (1) and (2) on the thrust and power is shown in Figs. $6 \mathrm{~d}$ and $\underline{6 \mathrm{e}}$. Figure $\overline{6} \mathrm{~d}$ indicates that both thrust and power decrease as the flap motion changes from a sine motion to a triangular wave. The variations of thrust and power for the range of $K_{\Phi}$ considered are less than $20 \%$. Both thrust and power increase when a square wave is used for the pitching motion, as shown in Fig. 6e. The increase in thrust $(35 \%)$ is larger than the increase in power (less than 20\%). Thus, a rapid pitch motion can be used to produce a significant increase in thrust with only a minor penalty on the power consumption. As shown in Fig. 6f, the power decreases by $15 \%$ when the location of the pitching axis is moved from the leading edge $(a=0)$ to the quarter-chord point $(a=0.25)$. However, the change in thrust is relatively small: $6 \%$. Therefore, pitching about the quarter-chord is beneficial for power consumption reduction with a relatively minor reduction in thrust. Both thrust and power increase monotonically with increase in flapping frequency, as shown in Fig. 6g.

Practical constraints can often limit the available power. Therefore, a constrained optimization study was conducted to maximize thrust for a specified amount of power. The power available was varied from the power required to generate maximum thrust, denoted by $P_{T_{\max }}$, to $0.5 P_{T_{\max }}$. The Pareto front corresponding to the maximum thrust for the specified value of power is shown in Fig. 7a. Note that the decrease in thrust is less than $30 \%$ of $T_{\max }$ for a $50 \%$ decrease in power. The true function evaluations at several points on the curve are represented by the squares in Fig. 7a. The relative errors between the surrogate predictions and the true function evaluations, plotted against the normalized value of the corresponding true function evaluation $\left(T / T_{\max }\right.$ or $\left.P / P_{T_{\max }}\right)$, are shown in Fig. $7 \mathrm{~b}$. The errors in thrust and power prediction, which are less than $18 \%$, are consistent with the errors listed in Tables 2 and 3 .

\section{B. Results for Flexible Wings}

An aeroelastic optimization study was conducted using anisotropic wings built from unstressed Capran film (membrane) supported by a carbon-fiber-based spar-rib skeleton. The wing configuration is depicted in Fig. 1a. The thicknesses of the leading-edge spar and the five ribs, shown in Fig. 1a, are used as the DV that control flexibility. The material properties of the membrane and composite skeleton, the widths of the leading-edge spar and the battens, and the thickness of the membrane are fixed [10] and are provided in Table 6 . The kinematic and structural parameters, which represent the DV for the flexible wings, together with their allowed ranges of variation are given in Table $\underline{7}$. 


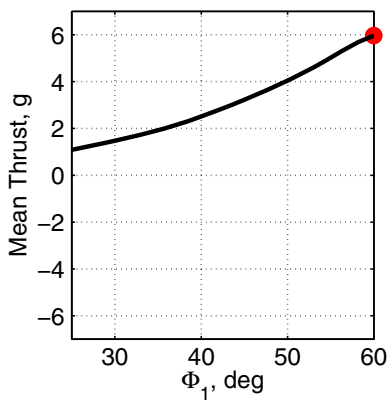

a) Flap amplitude

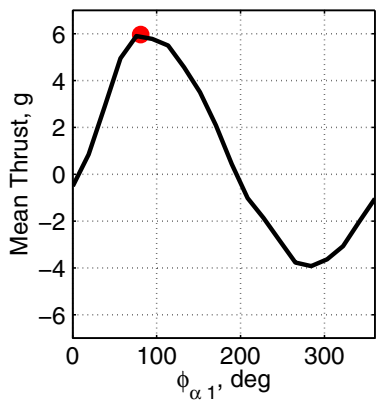

c) Phase angle
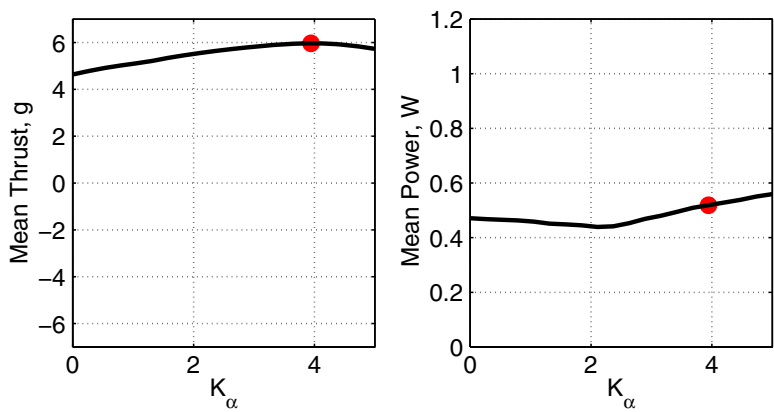

e) Shape parameter for pitch motion

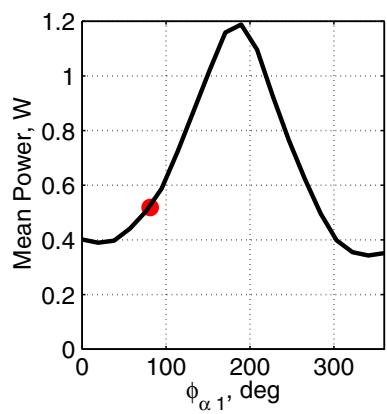

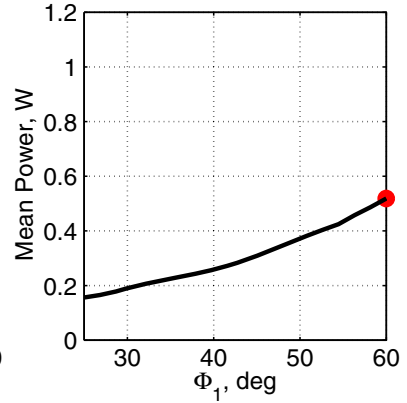
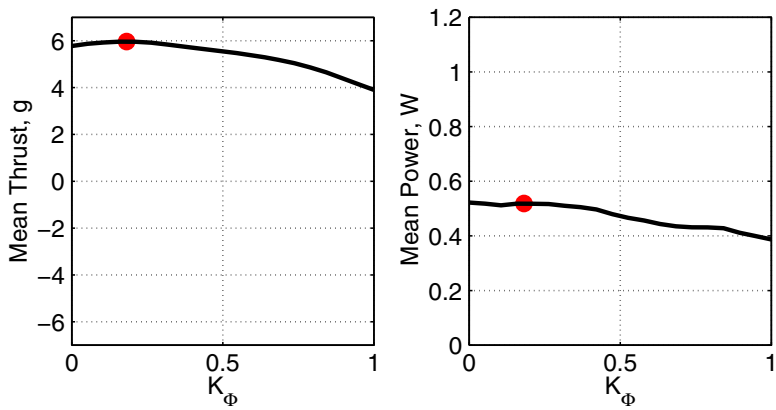

d) Shape parameter for flap motion
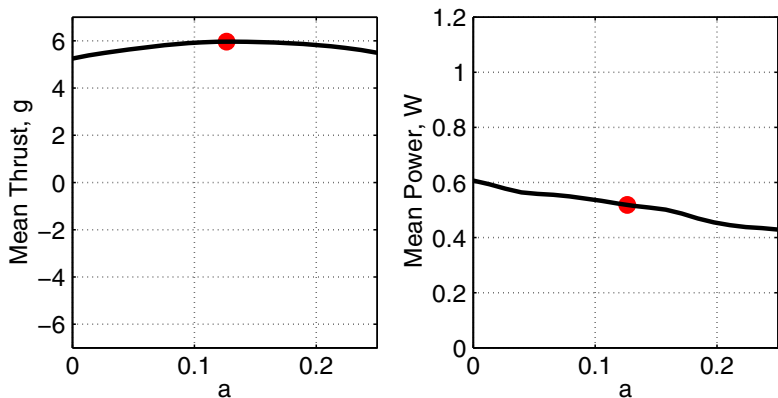

f) Feathering axis offset

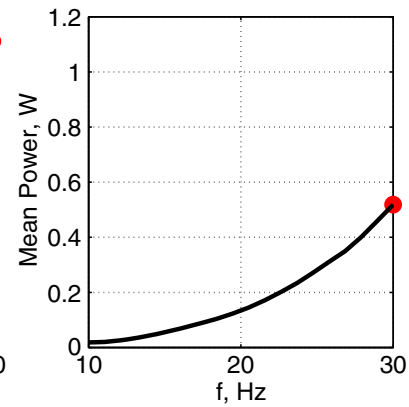

g) Flapping frequency

Fig. 6 Variation of thrust and power with kinematic design variables for rigid wings.

The finite-element model of the wing, shown in Fig. 1a, is composed of 765 shell elements (element type 75 in MARC). Wing kinematics are implemented as displacement boundary conditions at the nodes that form the triangular root. During the simulations, a pressure-based filter [22], described by Eq. (7), was used to limit the magnitude of numerical noise that is transmitted to the flexible wing:

$$
p_{\text {app }}=\frac{p_{\text {comp }}}{\left|p_{\text {comp }}\right|} \min \left\{\left|p_{\text {comp }}\right|,\left|p_{\text {limit }}\right|\right\}
$$

where $|\cdot|$ denotes the absolute value or magnitude, and $p_{\text {comp }}, p_{\text {limit }}$, and $p_{\text {app }}$ are the computed, applied, and limit values of aerodynamic pressure, respectively. For the cases considered, $p_{\text {limit }}=81 p_{\text {ref }}$, where the reference pressure is given by $p_{\text {ref }}=\frac{1}{2} \rho_{\infty} U_{\text {tip }}^{2}$ and $U_{\text {tip }}=2 \pi f \beta_{0} R_{\text {span }}$.

The kriging surrogates were created initially from a set of 199 points using an OLH sampling of the parameter space described in Table 7. The accuracy of the surrogates was tested at 49 points, also obtained by OLH sampling of the parameter space described in Table 7. Each simulation for generating the fitting and testing data was conducted using a single $2.53 \mathrm{GHz}$ Intel Xeon E5540 processor (4 GB RAM). The maximum time per simulation was $15 \mathrm{~h}$. The aerodynamic calculations for the flexible wings were not parallelized due to restrictions associated with MARC. Thus, the data for each sample point were computed using a single processor. The accuracy of the surrogates was first improved by 372 additional fitting points 


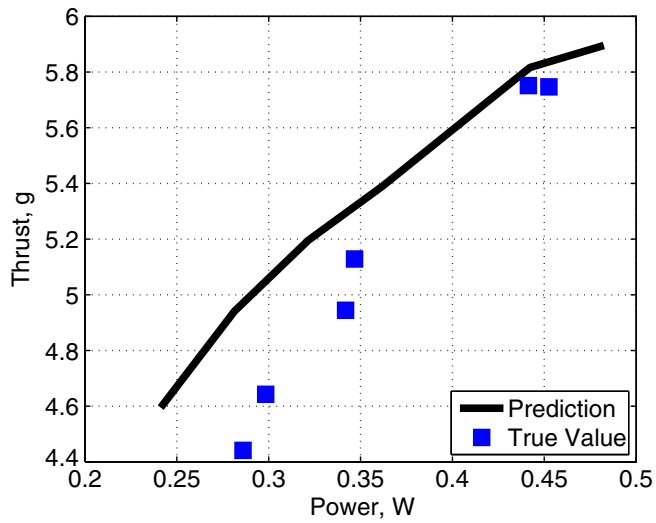

a) Maximum thrust for specified power

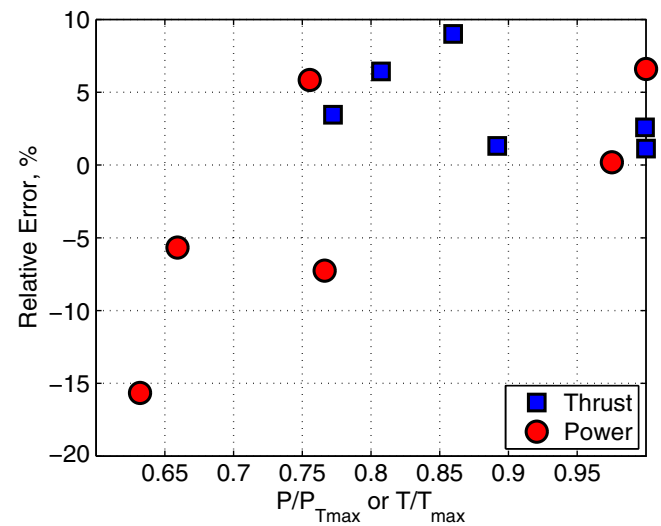

b) Errors in prediction of thrust and power

Fig. 7 Constrained optimization of rigid wings.

Table 6 Material properties of the composite skeleton and membrane

\begin{tabular}{lc}
\hline \hline Parameter & Composite \\
\hline & $233 \mathrm{GPa}$ \\
$E_{11}$ & $23.1 \mathrm{GPa}$ \\
$E_{22}$ & $10.5 \mathrm{GPa}$ \\
$E_{12}$ & 0.05 \\
$\nu_{12}$ & $1740 \mathrm{~kg} / \mathrm{m}^{3}$ \\
$\rho$ & Membrane \\
\hline$E$ & $2.76 \mathrm{GPa}$ \\
$\nu_{12}$ & 0.489 (Incompressible) \\
$\rho$ & $1384 \mathrm{~kg} / \mathrm{m}^{3}$ \\
Thickness & $15 \mathrm{microns}$ \\
\hline \hline
\end{tabular}

identified using EGO. These points were determined in four cycles, where the surrogate was used in EGO and refitted by including the new points for the next cycle. Significant errors at the boundary corresponding to $\left\{\Phi_{1}=55 \mathrm{deg}, f=25 \mathrm{~Hz}\right\}$ were noted. Therefore, 720 additional points were included using a combination of EGO and OLH sampling. First, two sets of OLH samplings were used. In the first set, 276 points were selected for the ranges given in Table 8 . In the second set, 99 points were selected for $\alpha_{1}=0 \mathrm{deg}$,

Table 9 Errors in thrust

\begin{tabular}{lcrr}
\hline \hline Errors & 199 points & 571 points & 1291 points \\
\hline \multicolumn{4}{c}{ Mean and maximum errors } \\
\hline Mean, \% & 10.5 & 4.5 & 4.4 \\
Maximum, \% & 43 & 19.3 & 15.5 \\
\multicolumn{4}{c}{ Distribution of errors } \\
\hline$>20 \%$ & 6 points & 0 points & 0 points \\
$<20 \%$ & 43 points & 49 points & 49 points \\
\hline \hline
\end{tabular}

Table 10 Errors in power

\begin{tabular}{lc}
\hline \hline Description & Bounds \\
\hline Flap amplitude & $10 \leq \Phi_{1} \leq 55 \mathrm{deg}$ \\
Pitch amplitude & $0 \leq \alpha_{1} \leq 50 \mathrm{deg}$ \\
Phase amplitude & $0 \leq \phi_{\alpha 1} \leq 360 \mathrm{deg}$ \\
Shape parameter in flap & $0 \leq K_{\Phi} \leq 0.8$ \\
Shape parameter in pitch & $0 \leq K_{\alpha} \leq 4$ \\
Location of pitch axis & $0 \leq a \leq 0.25 c_{r}$ \\
Flapping frequency, Hz & $10 \leq f \leq 25 \mathrm{~Hz}$ \\
Thickness of LE spar & $0.15 \leq T_{\mathrm{LE} \mathrm{spar}} \leq 0.4 \mathrm{~mm}$ \\
Thickness of rib 1 & $0.2 \leq t_{\text {rib1 }} \leq 0.3$ \\
Thickness of rib 2 & $0.05 \leq t_{\text {rib2 }} \leq 0.3 \mathrm{~mm}$ \\
Thickness of rib 3 & $0.05 \leq t_{\text {rib3 }} \leq 0.3 \mathrm{~mm}$ \\
Thickness of rib 4 & $0.05 \leq t_{\text {rib4 }} \leq 0.3 \mathrm{~mm}$ \\
Thickness of rib 5 & $0.05 \leq t_{\text {rib5 }} \leq 0.3 \mathrm{~mm}$ \\
\hline \hline
\end{tabular}

Table 8 Reduced bounds for the design variables

\begin{tabular}{|c|c|}
\hline Description & Bounds \\
\hline Flap amplitude & $52.5 \leq \Phi_{1} \leq 57.5 \mathrm{deg}$ \\
\hline Pitch amplitude & Same as in Table 5 \\
\hline Phase amplitude & Same as in Table $\overline{5}$ \\
\hline Shape parameter in flap & Same as in Table $\overline{5}$ \\
\hline Shape parameter in pitch & Same as in Table $\overline{5}$ \\
\hline Location of pitch axis & Same as in Table $\overline{5}$ \\
\hline Flapping frequency & $22.5 \leq f \leq 27.5 \overline{\mathrm{Hz}}$ \\
\hline Thickness of LE spar & Same as in Table 5 \\
\hline Thickness of rib 1 & $0.05 \leq t_{\mathrm{rib} 1} \leq 0.3 \overline{\mathrm{mm}}$ \\
\hline Thickness of rib 2 & $0.025 \leq t_{\mathrm{rib} 2} \leq 0.3 \mathrm{~mm}$ \\
\hline Thickness of rib 3 & $0.025 \leq t_{\mathrm{rib} 3} \leq 0.3 \mathrm{~mm}$ \\
\hline Thickness of rib 4 & $0.025 \leq t_{\mathrm{rib} 4} \leq 0.3 \mathrm{~mm}$ \\
\hline Thickness of rib 5 & $0.025 \leq t_{\mathrm{rib} 5} \leq 0.3 \mathrm{~mm}$ \\
\hline
\end{tabular}

\begin{tabular}{lccc}
\hline \hline Errors & 199 points & 571 points & 1291 points \\
\hline \multicolumn{4}{c}{ Mean and maximum errors } \\
\hline Mean, \% & 6.6 & 5.6 & 2.6 \\
Maximum, \% & 22 & 30 & 21 \\
& Distribution of errors & \\
\hline$>20 \%$ & 2 points & 1 point & 1 point \\
$<20 \%$ & 47 points & 48 points & 48 points \\
\hline \hline
\end{tabular}

Table 11 Kinematic and structural variables corresponding to maximum thrust

\begin{tabular}{lcl}
\hline \hline Description & Rigid wing & Flexible wing \\
\hline$\Phi_{1}$, deg & 55 & 55 \\
$\alpha_{1}$, deg & 50 & 34.8 \\
$\phi_{\alpha 1}$, deg & 80.3 & 81.9 \\
$K_{\Phi}$ & 0.19 & 0.63 \\
$K_{\alpha}$ & 4 & 3.75 \\
$a$ & 0.18 & 0.23 \\
$f, \mathrm{~Hz}$ & 25 & 25 \\
$t_{\mathrm{LE} \mathrm{spar}}$ & & $0.3034 \mathrm{~mm}$ \\
$t_{\text {rib1 }}$ & & $0.2241 \mathrm{~mm}$ \\
$t_{\text {rib2 }}$ & & $0.1378 \mathrm{~mm}$ \\
$t_{\text {rib3 }}$ & & $0.2262 \mathrm{~mm}$ \\
$t_{\text {rib4 }}$ & & $0.1093 \mathrm{~mm}$ \\
$t_{\text {rib5 }}$ (prediction), g & 3.74 & $0.2100 \mathrm{~mm}$ \\
$T_{\text {max }}$ (pretion, g & 3.28 & 3.99 \\
True evaluation, & & 0.273 \\
$P_{T_{\text {max }}}$ (prediction), W & 0.215 & 0.272 \\
True evaluation, W & 0.198 & \\
\hline \hline
\end{tabular}


where the ranges of the remaining DV are given in Table 7. Next, 169 points were selected using EGO for $50 \leq \Phi_{1} \leq \overline{55} \mathrm{deg}$ and $20 \leq f \leq 25 \mathrm{~Hz}$, where the ranges of remaining DV are given in Table 7. Finally, 176 points were selected using OLH sampling for $\Phi_{1}=\overline{5} 5 \mathrm{deg}$ and $f=25 \mathrm{~Hz}$, where the ranges of remaining DV are given in Table $\mathbf{7}$. The additional points resulted in a decrease of the mean and maximum errors in both power and thrust. The magnitude and distribution of errors in the surrogates are summarized in Tables 9 and 10 . It is evident that the final surrogates have acceptable accuracy (errors less than 20\%) over a large region of the parameter space.
The kinematic and structural parameters that produce maximum thrust are presented in Table 11. The corresponding parameters for rigid wings are also provided. The true function evaluations for thrust yields $3.28 \mathrm{~g}$ for rigid wings and $3.99 \mathrm{~g}$ for flexible wings. The corresponding evaluations for power yield 0.198 and $0.272 \mathrm{~W}$ for rigid and flexible wings, respectively. This implies that the best flexible configuration generates $22 \%$ more thrust and consumes $37 \%$ more power compared to the best rigid wing. For the flexible wings, the maximum thrust in the set of fitting points is $3.9 \mathrm{~g}$, which is less than the corresponding $T_{\max }$ in Table 11 . Thus, the optimal flexible configuration predicted using the surrogate-based optimization

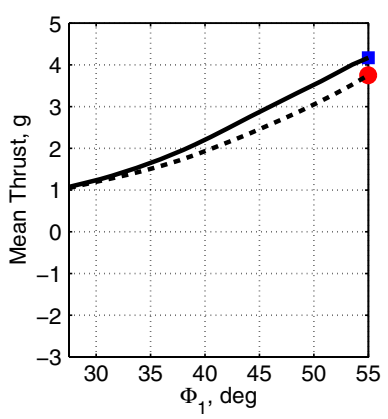

a) Flap amplitude

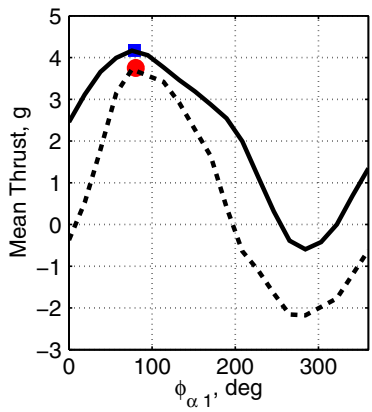

c) Phase angle

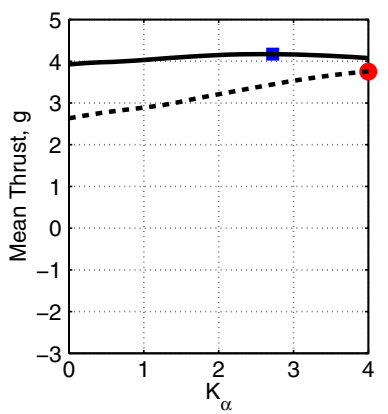

e) Shape parameter for pitch motion
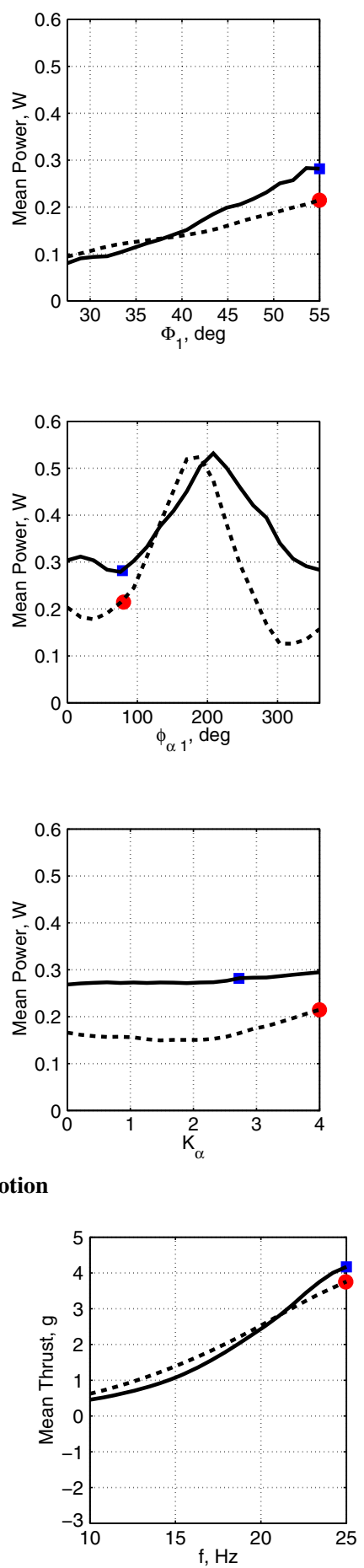

g) Flapping frequency
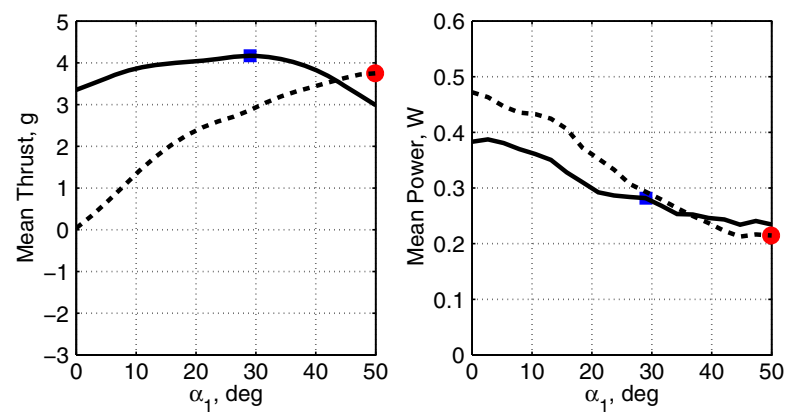

b) Pitch amplitude
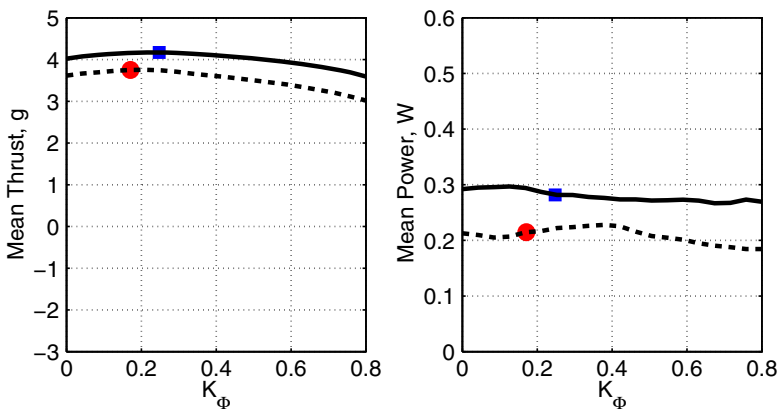

d) Shape parameter for flap motion
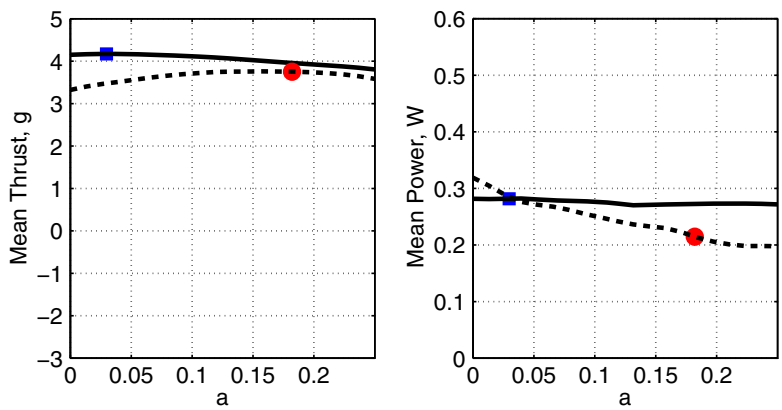

f) Pitch axis offset

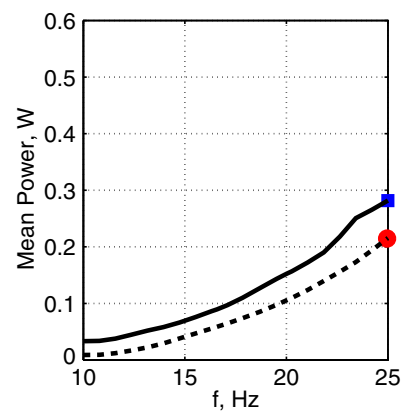

Fig. 8 Variation of thrust and power with kinematic parameters for flexible wings (solid line) and rigid wings (dashed line). 
approach produces higher thrust compared to the best thrustproducing design in the set of fitting points.

The variation of thrust and power with kinematic and structural parameters, calculated at the point of maximum thrust, are shown in Figs. 8 and 9 , respectively. Several similarities to the variation of thrust and power obtained for rigid wings (shown using the dashed line) are noted. Both the thrust and power increase monotonically with increase in flap amplitude, as shown in Fig. 8a. Figure $8 \mathrm{~b}$ indicates that thrust and power are nonmonotonic with pitch amplitude, suggesting that only a limited amount of pitching motion is useful. The best flexible configuration generates greater thrust compared to rigid wings while requiring a smaller amount of pitch. This is due to the twist produced that modifies the pitch distribution. Both thrust and power vary significantly over the range of phase angles considered, as shown in Fig. 8c. The variation of thrust with phase angle for flexible wings is similar to its variation for rigid wings. The variation of power is less significant. This suggests that the phase difference between flap and pitch motions for flexible wings can be used to tailor the thrust for fixed-stroke amplitudes and frequency.

The effect of the shape parameters on the thrust and power are shown in Figs. $8 \mathrm{~d}$ and $8 \mathrm{e}$. Figure $8 \mathrm{~d}$ indicates that the thrust increases slightly as the flap motion changes from a cosine motion to a triangular wave. The power variation is minor compared to the reduction in thrust, indicating that flap motion based on a triangular wave is beneficial. For flexible wings, the thrust and power vary only slightly with $K_{\alpha}$, as shown in Fig. 8e. This implies that a rapid pitch motion at the end of the flapping stroke has a greater impact on rigidwing performance when compared to flexible wings. The thrust and power decrease slightly as the pitching axis is moved from the leading edge of the flexible wing to the quarter-chord point. Thus, pitching about the quarter-chord point is beneficial. The opposite trend is found for thrust generated by rigid wings, where it is beneficial to pitch about a point closer to the leading edge.

Both thrust and power increase with increasing flapping frequency, as shown in Fig. 8g. The flexible wings generate more thrust compared to rigid wings only for $f>22 \mathrm{~Hz}$. Below $22 \mathrm{~Hz}$, rigid wings produce slightly greater thrust for equal or less required power compared to flexible wings. Therefore, increasing wing flexibility in this region of the design space results in thrust reduction accompanied by a small increase in power required.

The variation of thrust and power with structural design variables are illustrated in Fig. 9. The thickness of the LE spar has a significant effect on both the thrust generated and power consumed by the flexible wings, as shown in Fig. 9a. The variation of thrust with $t_{\text {lespar }}$ is nonmonotonic, suggesting that only a limited amount of spanwise stiffness is beneficial. Both thrust and power increase with increase in $t_{\text {lespar }}$, implying that increasing wing stiffness enhances thrust production but also increases the power penalty. The variation of thrust and power with the thickness of ribs 1, 2, 3, and 4, shown in Figs. 9b-9e, is minor. Rib 5 affects the tip twist. The trends

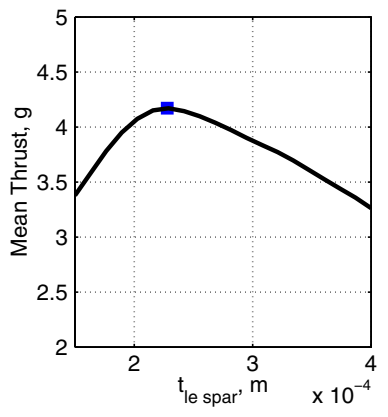

a) Thickness of LE spar

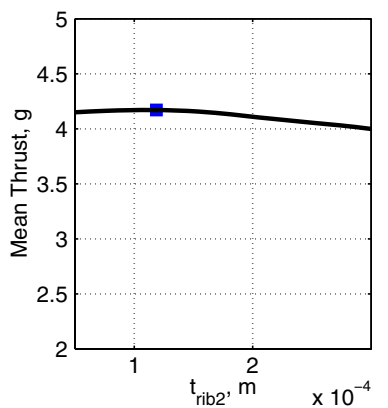

c) Thickness of rib 2

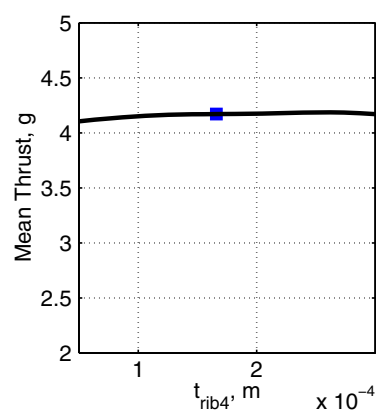

e) Thickness of rib 4
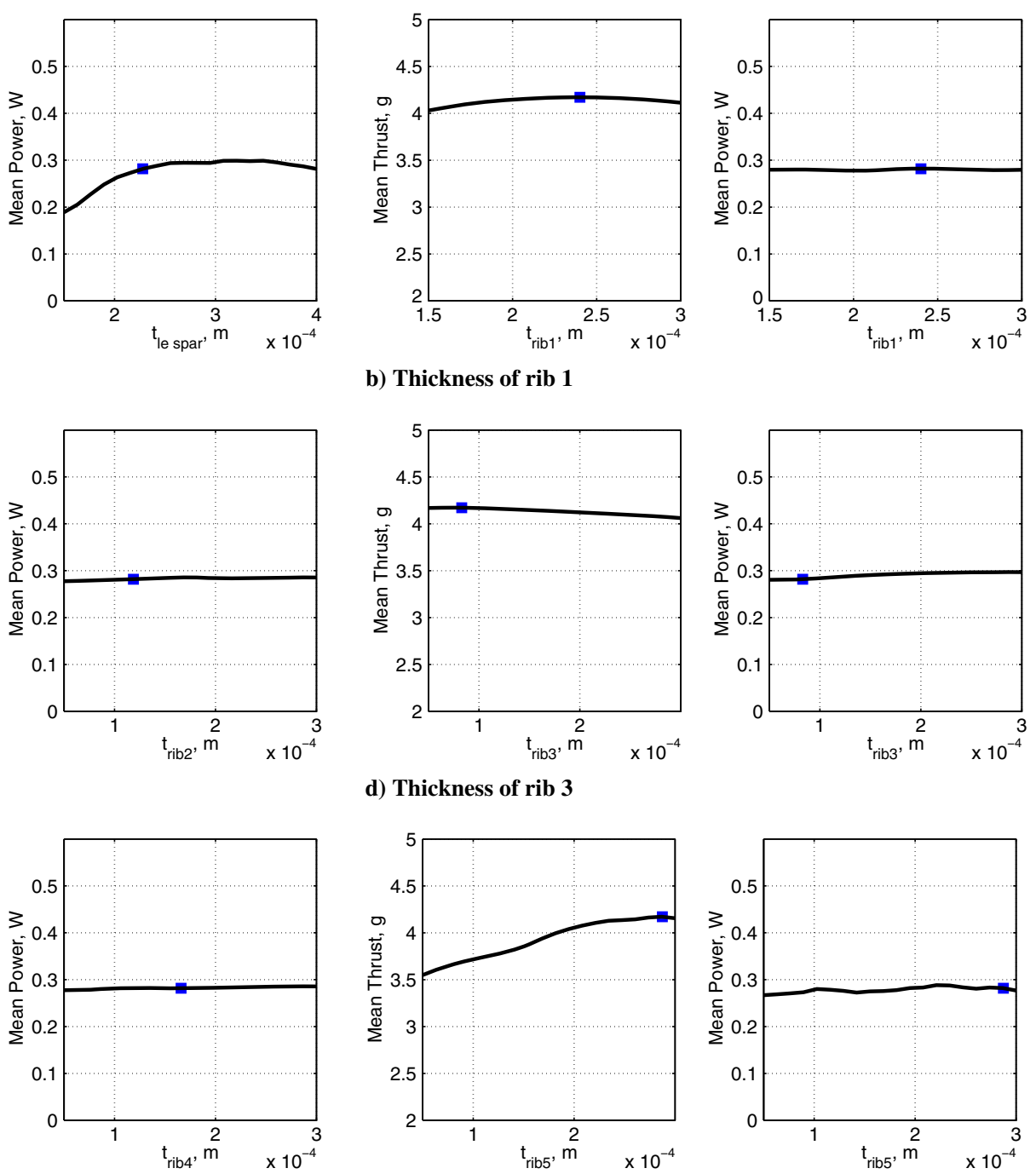

b) Thickness of rib 1
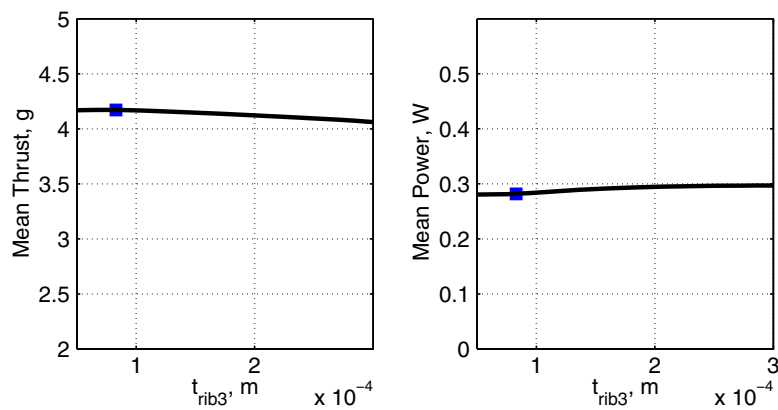

d) Thickness of rib 3
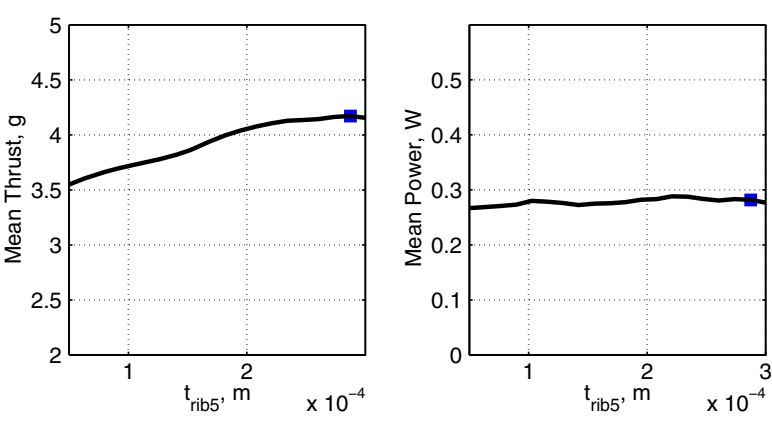

f) Thickness of rib 5

Fig. 9 Variation of thrust and power with structural parameters. 


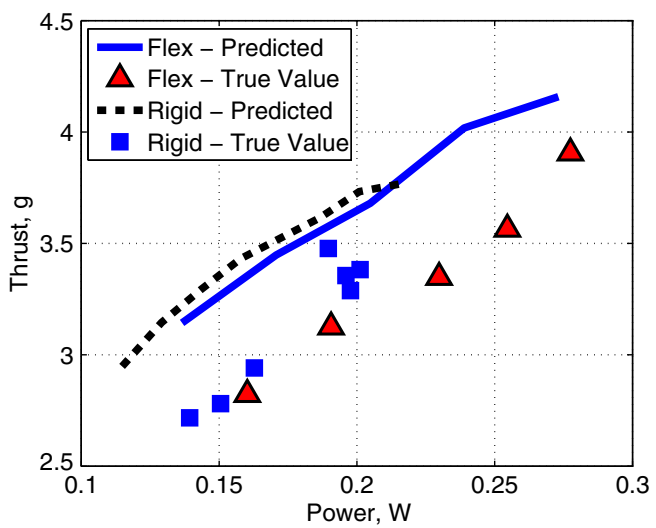

a) Maximum thrust for specified power

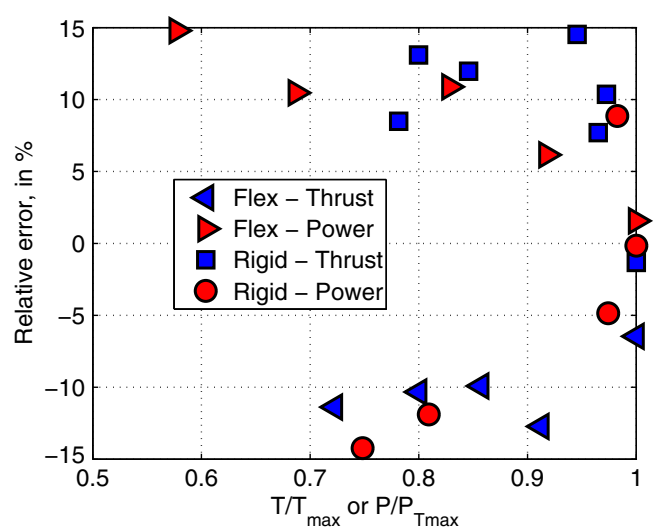

b) Errors in prediction of thrust and power

Fig. 10 Constrained optimization of rigid and flexible wings.

in Fig. 9f suggest that a stiffer chord on the outboard sections increases the thrust produced with only a minor increase in power penalty.

Results of a constrained optimization, in which the available power is specified as a fraction of the $P_{T_{\max }}$, are shown in Fig. 10a. The corresponding results obtained for rigid wings for the range of kinematic variables listed in Table $\underline{7}$ are shown. True function evaluations at several points on the curves are also shown. The best flexible configuration predicted by the surrogates produces $12 \%$ more thrust and requires $27 \%$ more power compared to the best rigid configuration. The true function evaluations show that the best flexible configuration produces $22 \%$ more thrust and requires $37 \%$ more power compared to the best rigid configuration. The surrogates predict that rigid wings generate greater thrust compared to flexible wings for a given required power. This trend is displayed by the true function evaluations that indicate that rigid configurations produced equal or slightly greater thrust compared to flexible configurations for the same value of power required. Thus, the surrogates adequately capture the trends in both thrust and power. Several flexible configurations produced equal or greater thrust compared to the best rigid configuration. However, they also required greater amounts of power.

The relative errors in prediction, plotted against the normalized value of the corresponding true function evaluation $\left(T / T_{\max }\right.$ or $\left.P / P_{T_{\max }}\right)$, are shown in Fig. 10b. The surrogates for both thrust and power have acceptable accuracy (errors less than or equal to $20 \%$ ). For both rigid and flexible wings, the errors in prediction of power increase as $P / P_{T_{\max }}$ decreases.

The propulsive efficiencies (thrust per unit power) of the rigid and flexible configurations identified in Fig. 10a are shown in Fig. 11. It is evident that the propulsive efficiency decreases as thrust increases for both rigid and flexible wings. The rigid wings have a 15-30\% higher

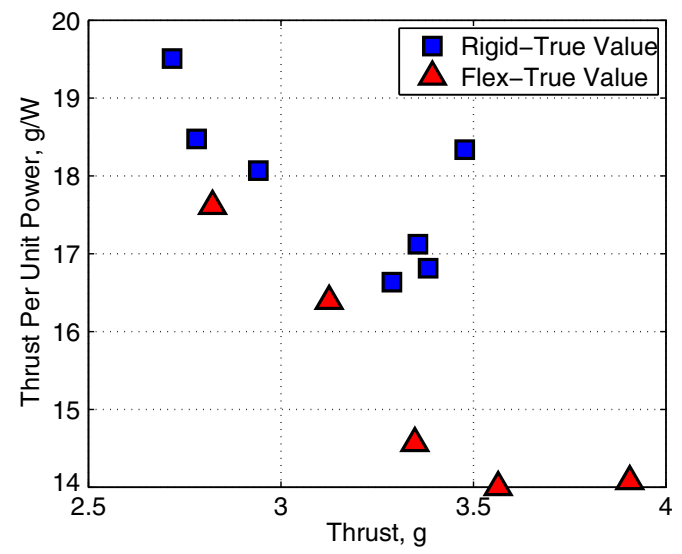

Fig. 11 Propulsive efficiency of the rigid and flexible configurations. propulsive efficiency compared to the flexible configurations when similar values of thrust are generated. The results in Figs. 10a and 11 indicate that wing flexibility is beneficial for thrust production but has a detrimental impact on the power consumption and propulsive efficiency. This suggests that the choice between rigid and flexible wings is likely to depend on the relative importance of thrust generation and propulsive efficiency for a particular wing design.

\section{Conclusions}

Surrogate-based optimization of rigid and flexible flapping wings in hover is described. The surrogate models, generated by kriging, are used to predict the time-averaged thrust and power by the wings. The accuracy of the surrogates was improved using a combination of the EGO approach combined with OLH sampling centered at the boundaries. The surrogates were used to examine the variation of thrust and power with the kinematic and structural parameters. Constrained optimization studies, in which the thrust was maximized for a specified amount of available power, were also conducted. The principal conclusions are provided next.

1) The surrogates, for both rigid and flexible wings, were effective for identifying configurations that were superior to the best configuration in the set of fitting points.

2) A rapid pitching motion at the end of the flapping stroke was beneficial for thrust production for both rigid and flexible wings.

3) The phase angle between flap and pitch motions, which modifies the wake capture, has a significant impact on the thrust generated and the power consumed by the wings. Therefore, the phase angle can be used to tailor wing performance for fixed-stroke amplitudes and flapping frequency.

4) Among all structural design variables, the thickness of the leading-edge spar had the greatest influence on the thrust generated and power consumed by flexible wings.

5) The peak performance achieved with flexible wings required a smaller amount of pitch actuation compared to rigid wings. This is due to the elastic twist produced in the flexible wings that modifies the effective angle of attack. Therefore, wing flexibility can be used as a passive means of enhancing performance while reducing the complexity of the wing actuation.

6) Several flexible configurations produced greater thrust when compared to the best thrust-producing rigid configuration. However, rigid wings had greater propulsive efficiency compared to flexible wings when similar levels of thrust were generated. The rigid wings were found to offer similar, and sometimes better, thrust for specified power compared to flexible wings in several regions of the parameter space. Thus, the choice between rigid and flexible wings depends on the relative importance of thrust and efficiency.

The wings examined in the current paper represent only a limited set of the large number of feasible planforms and wing constructions (spar batten, bio-inspired, etc.). The current work suggests that the preference between rigid and flexible wings is likely to depend on the 
number of design variables and range of parameters considered. This motivates the need to consider combined optimization of wing planform, construction, kinematics, and structural properties.

\section{Acknowledgments}

This work was supported by the U.S. Air Force Office of Scientific Research's Multidisciplinary University Research Initiative with Douglas R. Smith as Program Director.

\section{References}

[1] Mueller, T. J., "Fixed and Flapping Wing Aerodynamics for Micro Air Vehicle Applications," Progress in Aeronautics and Astronautics, Vol. 195, AIAA, Reston, VA, 2001, pp. 1-13, Chap. 1.

[2] Sane, S. P., "The Aerodynamics of Insect Flight," Journal of Experimental Biology, Vol. 206, Dec. 2003, pp. 4191-4208. doi: $10.1242 / \mathrm{jeb} .00663$

[3] Shyy, W., Lian, Y., Tang, J., Viieru, D., and Liu, H., Aerodynamics of Low Reynolds Number Flyers, Cambridge Univ. Press, New York, 2008, pp. 1-27, 101-158, Chaps. 1, 4

[4] Shyy, W., Aono, H., Chimakurthi, S., Trizila, P., Kang, C.-K., Cesnik, C., and Liu, H., "Recent Progress in Flapping Wing Aerodynamics and Aeroelasticity," Progress in Aerospace Sciences, Vol. 46, No. 7, 2010, pp. 284-327. doi:10.1016/j.paerosci.2010.01.001

[5] Aono, H., Liang, F., and Liu, H., "Near- and Far-Field Aerodynamics in Insect Hovering Flight: An Integrated Computational Study," Journal of Experimental Biology, Vol. 211, No. 2, 2008, pp. 239-257. doi:10.1242/jeb.008649

[6] Keennon, M., Klingebiel, K., Won, H., and Andriukov, A., "Development of the Nano Hummingbird: A Tailless Flapping Wing Micro Air Vehicle," 50th AIAA Aerospace Sciences Meeting, AIAA Paper 2012-0588, Jan. 2012.

[7] Berman, G. J., and Wang, Z. J., "Energy-Minimizing Kinematics in Hovering Insect Flight," Journal of Fluid Mechanics, Vol. 582, July 2007 , pp. 153-168. doi:10.1017/S0022112007006209

[8] Tuncer, I. H., and Kaya, M., "Optimization of Flapping Airfoils for Maximum Thrust and Propulsive Efficiency," AIAA Journal, Vol. 43, No. 11, 2005, pp. 2329-2336. doi:10.2514/1.816

[9] Stanford, B., Kurdi, M., Beran, P., and McClung, A., "Shape, Structure, and Kinematic Parameterization of a Power-Optimal Hovering Wing," 51st AIAA/ASME/ASCE/AHS/ASC Structures, Structural Dynamics, and Materials Conference, AIAA Paper 2010-2963, April 2010.

[10] Gogulapati, A., Friedmann, P. P., Kheng, E., and Shyy, W., "Approximate Aeroelastic Modeling of Flapping Wings in Hover," AIAA Journal, Vol. 51, No. 3, March 2013, pp. 1-17. doi:10.2514/1.J051801

[11] Gogulapati, A., and Friedmann, P. P., "Approximate Aerodynamic and Aeroelastic Modeling of Flapping Wings in Forward Flight," AIAA Journal, Vol. 52, No. 1, Jan. 2014, pp. 212-218. doi:10.2514/1.J052596

[12] Vandenheede, R. B. R., Bernal, L. P., Morrison, C. L., Gogulapati, A., Friedmann, P. P., Kang, C. K., and Shyy, W., "Experimental and Computational Study on Flapping Wings with Bio-Inspired Hover Kinematics," AIAA Journal, Vol. 52, No. 5, May 2014, pp. 1047-1058. doi:10.2514/1.J052644

[13] Queipo, N., Haftka, R., Shyy, W., Goel, T., Vaidyanathan, R., and Kevin Tucker, P., "Surrogate-Based Analysis and Optimization," Progress in Aerospace Sciences, Vol. 41, No. 1, 2005, pp. 1-28. doi:10.1016/j.paerosci.2005.02.001

[14] Trizila, P., Kang, C.-K., Visbal, M., and Shyy, W., "Unsteady Fluid Physics and Surrogate Modeling of Low Reynolds Number, Flapping Airfoils," 38th AIAA Fluid Dynamics Conference and Exhibit, AIAA Paper 2008-3821, June 2008.

[15] Glaz, B., Friedmann, P. P., and Liu, L., "Surrogate Based Optimization of Helicopter Rotor Blades for Vibration Reduction in Forward Flight," Structural and Multidisciplinary Optimization, Vol. 35, No. 4, 2008, pp. 341-363. doi:10.1007/s00158-007-0137-z

[16] Glaz, B., Friedmann, P., and Liu, L., "Helicopter Vibration Reduction Throughout the Entire Flight Envelope Using Surrogate-Based Optimization," Journal of the American Helicopter Society, Vol. 54, No. 1, 2009, Paper 012007. doi:10.4050/JAHS.54.012007
[17] MSC. MARC, Vols. A-D, Software Package, MSC Software Corp., Newport Beach, CA, 2005, www.mscsoftware.com [retrieved 6 June 2014].

[18] Ansari, S. A., Żbikowski, R., and Knowles, K., "Non-Linear Unsteady Aerodynamic Model for Insect-Like Flapping Wing in the Hover. Part 1: Methodology and Analysis," Proceedings of the Institution of Mechanical Engineers, Part G: Journal of Aerospace Engineering, Vol. 220, No. 2, 2006, pp. 61-83. doi:10.1243/09544100JAERO49

[19] Ansari, S. A., Żbikowski, R., and Knowles, K., "Non-Linear Unsteady Aerodynamic Model for Insect-Like Flapping Wing in the Hover. Part 2: Implementation and Validation," Proceedings of the Institution of Mechanical Engineers, Part G: Journal of Aerospace Engineering, Vol. 220, No. 3, 2006, pp. 169-186. doi:10.1243/09544100JAERO50

[20] Bathe, K., Ramm, E., and Wilson, E. L., "Finite Element Formulations for Large Deformation Dynamic Analysis," International Journal for Numerical Methods in Engineering, Vol. 9, 1975, pp. 353-386. doi:10.1002/(ISSN)1097-0207

[21] Chung, J., and Hulbert, G. M., "A Family of Single-Step Houbolt Time Integration Algorithms for Structural Dynamics," Computational Methods in Applied Mechanics and Engineering, Vol. 118, Nos. 1-2, 1994, pp. 1-11. doi:10.1016/0045-7825(94)90103-1

[22] Gogulapati, A., "Nonlinear Approximate Aeroelastic Analysis of Flapping Wings in Hover and Forward Flight," Ph.D. Thesis, Univ. of Michigan, Ann Arbor, MI, 2011.

[23] Forrester, A. I. J., Sóbester, A., and Keane, A. J., Engineering Design via Surrogate Modelling: A Practical Guide, Wiley, Hoboken, NJ, 2008.

[24] Simpson, T. W., Poplinski, J. D., Koch, P. N., and Allen, J. K., "Metamodels for Computer-Based Engineering Design: Survey and Recommendations," Engineering with Computers, Vol. 17, No. 2, 2001, pp. 129-150. doi:10.1007/PL00007198

[25] Lophaven, S. N., Nielsen, H. B., and Sondergaard, J., "A MATLAB Kriging Toolbox, Version 2.0," Technical Univ. of Denmark TR-IMMTR-2002-12, Dept. of Informatics and Mathematical Modeling, Kongens Lyngby, Denmark, 2002, http://www.imm.dtu.dk/ hbni/dace/ [retrieved 6 June 2014].

[26] Jones, D. R., Schonlau, M., and Welch, W. J., "Efficient Global Optimization of Expensive Black-Box Functions," Journal of Global Optimization, Vol. 13, No. 4, 1998, pp. 455-492. doi:10.1023/A:1008306431147

[27] Jones, D. R., "A Taxonomy of Global Optimization Methods Based on Response Surfaces," Journal of Global Optimization, Vol. 21, No. 4, 2001, pp. 345-383. doi:10.1023/A:1012771025575

[28] Jin, R., Chen, W., and Sudjianto, A., "An Efficient Algorithm for Constructing Optimal Design of Computer Experiments," Journal of Statistical Planning and Inference, Vol. 134, No. 1, 2005, pp. 268 287. doi:1016/j.jspi.2004.02.014

[29] Koch, P. N., Evans, J. P., and Powell, D., "Interdigitation for Effective Design Space Exploration Using iSIGHT," Structural and Multidisciplinary Optimization, Vol. 23, No. 2, 2002, pp. 111-126. doi:10.1007/s00158-002-0171-9

[30] Price, K. V., Storn, R. M., and Lampinen, J. A., Differential Evolution: A Practical Approach to Global Optimization, Springer-Verlag, Berlin, 2005, pp. 37-134, Chap. 2.

[31] Whitley, D., "A Genetic Algorithm Tutorial," Statistics and Computing, Vol. 4, No. 2, 1994, pp. 65-85. doi:10.1007/BF00175354

[32] Eberhart, R., and Kennedy, J., "A New Optimizer Using Particle Swarm Theory," Proceedings of the 6th International Symposium on Micro Machine and Human Science, IEEE Publ., Piscataway, NJ, Oct. 1995, pp. 39-43.

[33] Spall, J. C., Introduction to Stochastic Search and Optimization: Estimation, Simulation, and Control, Vol. 65, Wiley-Interscience Series in Discrete Mathematics and Optimization, Wiley, Hoboken, NJ, 2005, pp. 208-227, Chap. 8.

[34] Byrd, R. H., Gilbert, J. C., and Nocedal, J., "A Trust Region Method Based on Interior Point Techniques for Nonlinear Programming," Mathematical Programming, Vol. 89, No. 1, 2000, pp. 149-185. doi:10.1007/PL00011391

[35] Byrd, R. H., Hribar, M. E., and Nocedal, J., "An Interior Point Algorithm for Large-Scale Nonlinear Programming," SIAM Journal on Optimization, Vol. 9, No. 4, 1999, pp. 877-900. doi: $10.1137 / \mathrm{S} 1052623497325107$ 
[36] Waltz, R. A., Morales, J. L., Nocedal, J., and Orban, D., "An Interior Algorithm For Nonlinear Optimization That Combines Line Search and Trust Region Steps," Mathematical Programming, Vol. 107, No. 3, 2006, pp. 391-408. doi:10.1007/s10107-004-0560-5

[37] Wu, P., Ifju, P., Stanford, B., Sallstrom, E., Ukeiley, L., Love, R., and Lind, R., "A Multidisciplinary Experimental Study of Flapping Wing
Aeroelasticity in Thrust Production," 50th AIAA/ASME/ASCE/AHS/ ASC Structures, Structural Dynamics, and Materials Conference, AIAA Paper 2009-2413, May 2009.

R. Haftka Associate Editor 